\title{
Experimental and Computational Analysis of Model-Support Interference in Low-Speed Wind-Tunnel Testing of Fuselage-Boundary-Layer Ingestion
}

\author{
Biagio Della Corte*, André A.V. Perpignan, Martijn van Sluis, and Arvind Gangoli Rao \\ Delft University of Technology, Faculty of Aerospace Engineering \\ Kluyverweg 1, 2628HS, Delft, The Netherlands
}

\begin{abstract}
Junction flow caused by the aerodynamic interaction between a wind-tunnel model and the support structure can largely influence the flowfield and hence the experimental results. This paper discusses a combined numerical and experimental study which was carried out to mitigate the model-support interference in a wind-tunnel test setup for the study of fuselage boundary-layer ingestion. The setup featured an axisymmetric fuselage mounted through a support beam, covered by a wing-shaped fairing. The junction flow appearing at the fuselage-fairing connection produced undesired flow distortions at the fuselage aft section, due to the formation of an horseshoe vortex structure at the fairing leading edge. Numerical and experimental analysis were performed with the aim of reducing the distortion intensity by improving the fairing design. Results show that modifying the leading-edge shape of the fairing effectively decreased the flowfield distortions. Moreover, the addition of a dummy fairing diametrically opposed to the first one was found to be beneficial due to the enhancement of the configuration symmetry.
\end{abstract}

\section{Nomenclature}

\section{Latin symbols}

$c \quad$ Wing chord length, [m]

$H \quad$ Boundary layer shape factor

$R \quad$ Maximum fuselage radius, [m]

$L \quad$ Fuselage length, [m]

$p_{T} \quad$ Total pressure, $[\mathrm{Pa}]$

$q_{\infty} \quad$ Freestream dynamic pressure, [Pa]

$r \quad$ Radial coordinate, [m]

$s \quad$ Wall-normal distance, $[\mathrm{m}]$

$u \quad$ Axial velocity component, $[\mathrm{m} / \mathrm{s}]$

$V_{\infty} \quad$ Freestream velocity, $[\mathrm{m} / \mathrm{s}]$

$X \quad$ Axial coordinate, $[\mathrm{m}]$

$X_{T E} \quad$ Axial distance from the fuselage trailing edge, [m]

$Y \quad$ Lateral coordinate, $[\mathrm{m}]$

$Z \quad$ Vertical coordinate, [m]

*e-mail: B.DellaCorte@tudelft.nl 


\section{Greek symbols}

$\delta \quad$ Boundary layer physical thickness, [m]

$\theta \quad$ Boundary layer momentum thickness, [m]

\section{Acronyms}

$\begin{array}{ll}\text { BLI } & \text { Boundary Layer Ingestion } \\ \text { CENTRELINE } & \text { ConcEpt validatioN sTudy foR fusElage wake-filLIng propulsioN intEgration } \\ \text { CFD } & \text { Computational Fluid Dynamics } \\ \text { EC } & \text { European Commission } \\ \text { LE } & \text { Leading edge } \\ \text { LTT } & \text { Low-Turbulence Wind-Tunnel } \\ \text { PFC } & \text { Propulsive Fuselage Concept } \\ \text { RSM } & \text { Reynolds Stress Model } \\ \text { SST } & \text { Shear Stress Transport } \\ \text { TE } & \text { Trailing edge }\end{array}$

\section{Introduction}

The aerodynamic interaction taking place between a wind-tunnel model and the support structure can have a large influence on the flowfield and hence on the resulting measurements. The disturbances introduced in the flowfield by the model support can be divided into two categories: 1) far-field effects, mainly due to inviscid interactions (e.g. induced velocity components), and 2) near-field effects, mainly due to viscid interactions between the support and the boundary layer of the model [1]. Junction flow is one example of the near-field interaction effects. Junction flow appears when an obstacle is immersed in the boundary layer flowing along a wall [2]. As a consequence of the adverse pressure field induced by the obstacle, flow reversal occurs on the wall from which the obstacle is protruding [3]. The onset of flow reversal leads to the formation of an horseshoe vortex structure and corner separation $[4,5]$. One way of mitigating the junction flow is by reducing the induced pressure gradient through the employment of a leading-edge fillet. The performance and the design of LE fillets have been vastly assessed in literature, also through CFD-based methods [6, 7]. Van Oudheusden et al. [8] proposed a design procedure based on the combination of a panel method for the solution of the potential flow and an integral method for the solution of the approaching boundary layer on the attachment line.

This paper discusses the wind-tunnel model-support interference encountered in the development of a test rig for the study of boundary layer ingestion (BLI) at Delft University of Technology. The work, performed under the EC-funded Horizon 2020 CENTRELINE project [9], is aimed at a detailed aerodynamic study of the "Propulsive Fuselage Concept" (PFC). The concept features a boundary-layer ingesting fan integrated at the aft fuselage section. The goal of the experiments is that of analysing the configuration through momentumand energy-based approaches. In particular, the scope of the work is to isolate the effects of BLI from other integration effects (e.g. inflow distortions due to the wings). Therefore, an idealized aircraft model featuring an axysimmetric fuselage was utilized in the experiments. The fuselage model was connected to the external balance through a support beam, which was covered with a wing-shaped fairing. Numerical and experimental analysis have been carried out to design the fairing with the goal of mitigating the flow distortions introduced by the support structure setup. 


\section{Methodology}

\subsection{Experimental Setup}

The experimental campaigns were carried out in the Low-Turbulence Wind-Tunnel (LTT) facilities of Delft University of Technology. This closed-circuit wind-tunnel features a closed test section with an $1.25 \mathrm{~m} \times 1.80 \mathrm{~m}$ octagonal cross-section. The tunnel is driven by a $525 \mathrm{~kW}$ electric fan and is capable of a maximum test speed of around $120 \mathrm{~m} \mathrm{~s}^{-1}$. The freestream turbulence level is reported to be between $0.015 \%$ at $20 \mathrm{~m} \mathrm{~s}^{-1}$ and $0.07 \%$ at $75 \mathrm{~m} \mathrm{~s}^{-1}$.

A sketch of the setup is presented in Figure 1. The model used in the experiments consisted of an axisymmetric body representative of an aircraft fuselage. The fuselage featured a maximum radius $R=80 \mathrm{~mm}$ and a length $L=1776 \mathrm{~mm}$. Turbulent boundary-layer flow was ensured with a zig-zag transition strip at $5 \%$ of the fuselage lentgth from the nose. The fuselage model was centered with the test section and connected to the external balance through a vertical support beam. A wing-shaped fairing was mounted around the support beam and bolted directly to the wind-tunnel ceiling.

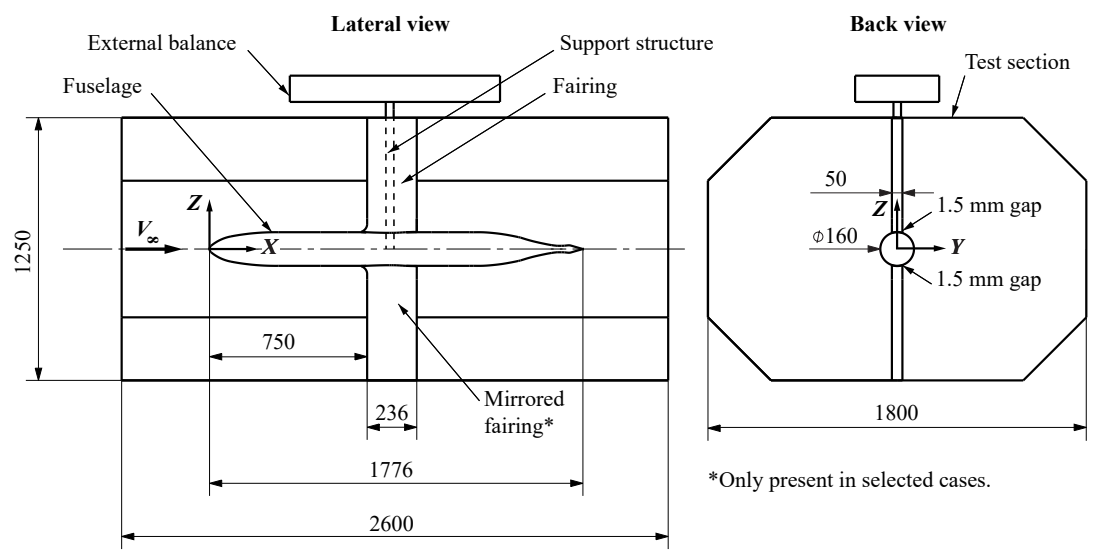

(a) Whole setup technical drawing.

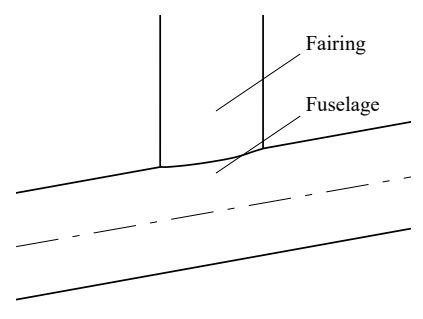

(b) Baseline configuration.

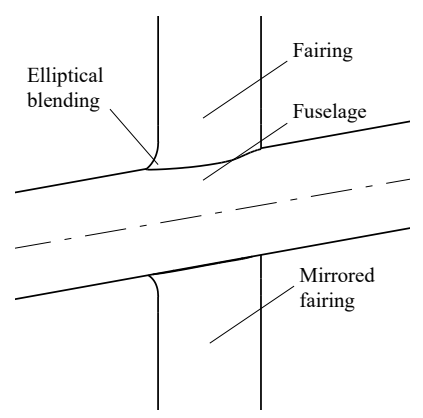

(c) Optimized configuration.

Figure 1: Experimental setup and different tested configurations. Dimensions in millimeters.

Two different fairing configurations, referred to as 'baseline' and 'optimized', were tested as sketched in Figures $1 \mathrm{~b}$ and $1 \mathrm{c}$. In both cases the fairings featured a NACA 66 4021 airfoil section and a rectangular planform with a chord $c=236 \mathrm{~mm}$. In the baseline configuration of 
Figure 1b, a single fairing without any fillet and a flat tip was implemented. A gap of around $1.5 \mathrm{~mm}$ was left between the fuselage and the fairing tip. On the contrary, the optimized configuration featured an elliptical leading-edge blending at the fuselage, as shown in Figure 1c. The tip of the fairing featured a curved surface with a curvature radius equal to the fuselage radius. The elliptical leading-edge fillet was obtained by following the design proposed by van Oudheusden et al. $[8,10]$ To minimize the distortions induced in the flowfield by the presence of the fairing, a second setup was obtained by mirroring the upper fairing around the horizontal plane and placed on the bottom part of the setup. All measurements were taken at zero-incidence conditions. The freestream velocity $V_{\infty}$ was set equal to $20 \mathrm{~m} \mathrm{~s}^{-1}$ and $40 \mathrm{~m} \mathrm{~s}^{-1}$.

Different measurement techniques were performed to quantify the airflow around the fuselage and the fairing surfaces. As already mentioned, an external six-component balance was used to measure the aerodynamic forces and moments acting on the fuselage surface. In addition, total pressure measurements were carried out in the wake of the model by means of a rake of 44 total-pressure probes. The scanned plane was orthogonal to the freestream velocity direction and placed at a distance from the trailing edge of the fuselage varying from $X_{T E}=1 R$ to $X_{T E}=2 R$. The probes were non-evenly distributed with a minimum lateral spacing of $3 \mathrm{~mm}$ (equal to $3.75 \%$ of $R$ ) at the center of the rake. The rake was traversed in the vertical (spanwise) direction with a spacing of $0.5 \mathrm{~mm}(0.625 \%$ of $R)$ at the fuselage center and gradually increasing at larger $Z$ positions. At each vertical position, measurements were taken with an electronic pressure scanner at $5 \mathrm{~Hz}$ for a period of $10 \mathrm{~s}$ and time-averaged to obtain the single data point.

\subsection{Numerical Setup}

In order to find a better option capable of reducing the interference of model support on the flow field, Computational Fluid Dynamics (CFD) simulations were carried out to investigate the performance of different fairing designs. As shown in Figure 2, in addition to the baseline (a), three new configurations were tested utilizing CFD, which featured a dummy fairing placed on the opposite side of the model (b), a fairing equipped with a leading edge insert (c), and a curved fairing tip following the model curvature (d). The CFD simulations were performed utilizing ANSYS Fluent. Computational meshes were unstructured and composed of tetrahedral elements with prism layers along the walls of the fuselage and fairing. The prism layers were composed of 20 layers with the total height sufficiently covering the boundary layers and the initial height in accordance with the adopted near-wall treatment $\left(\mathrm{y}^{+}\right.$in the order of 1). Three different turbulence models were employed: Spalart-Allmaras [11], $\kappa-\omega$ SST [12], and an $\omega$-based Reynolds Stress model [13]. Second order discretization was employed for all simulations. The SIMPLE pressure-velocity coupling scheme was adopted. Velocity was imposed to the inlet boundary $\left(40 \mathrm{~m} \mathrm{~s}^{-1}\right)$ along with $5 \%$ turbulence intensity and a turbulent viscosity ratio of 10 . The outlet of the domain was modelled by imposing a zero gauge pressure. A symmetry boundary condition was applied to all simulations with respect to the plane cutting through the centreline of the fuselage and fairing. The case with a mirrored fairing had another symmetry plane so that one fourth of the domain was simulated. The mesh independence study was conducted by refining the element sizes on the surface of the fuselage and fairing, as well as the elements in the surroundings of the fuselage, the fairing and their wakes. The prism layer settings were maintained for all meshes. Meshes with approximately 7, 13, 15 and 17 million elements were tested after which a mesh with 15 million elements was considered to be the best compromise between accuracy and computational time. 


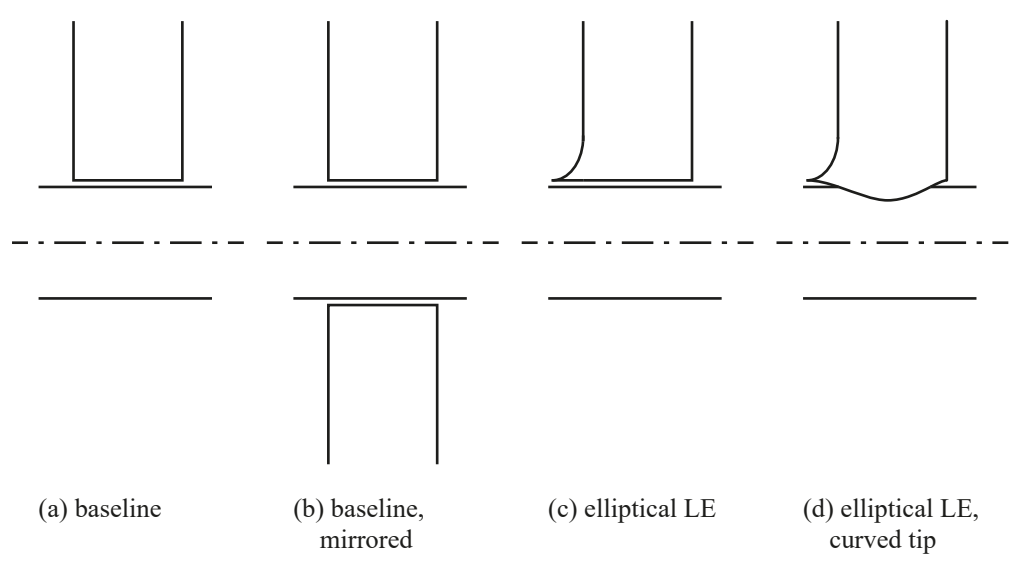

Figure 2: Comparison of the different fairing design iterations.
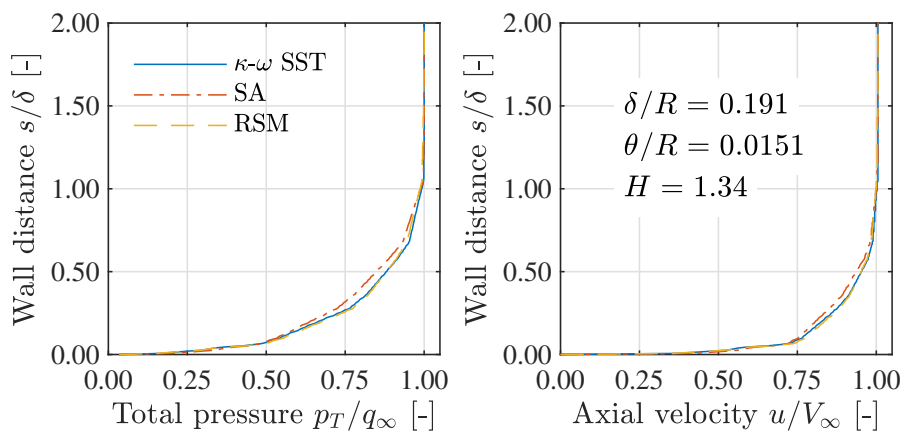

Figure 3: Total pressure and axial velocity profiles in the fuselage boundary layer measured $0.5 c$ upstream of the fairing leading edge.

\section{Results}

\subsection{CFD analysis}

The comparison between the three different turbulence models (Figures 3 and 4) show that they have similar performance. The near-wall total pressure and velocity profiles (Figure 3 ) show minor differences between the models. Additionally, the total pressure profiles at the measurement plane (Figure 4) show that all three models deviate from the experimental values on a similar fashion. Based on these results, the study of alternative configurations was performed utilizing the Spalart-Allmaras model, since its computational cost is lower.

The comparison between the four different configurations is shown on Figure 5. Some conclusions could be drawn from analysing these results: i) having a mirrored fairing (configuration b) decreases the intensity of the distortion and, obviously, creates another symmetry plane, ii) modifying the fairing LE (configuration c) provides only marginal improvement with respect to the baseline, and iii) having the fairing to follow the fuselage geometry on its tip reduces the distortion both in intensity as well as in its area. Therefore, these results led to the decision of adopting the optimized configuration (Figure 1), as it combines the benefits of having the mirrored fairing with the smoothed fairing and fuselage connection. 

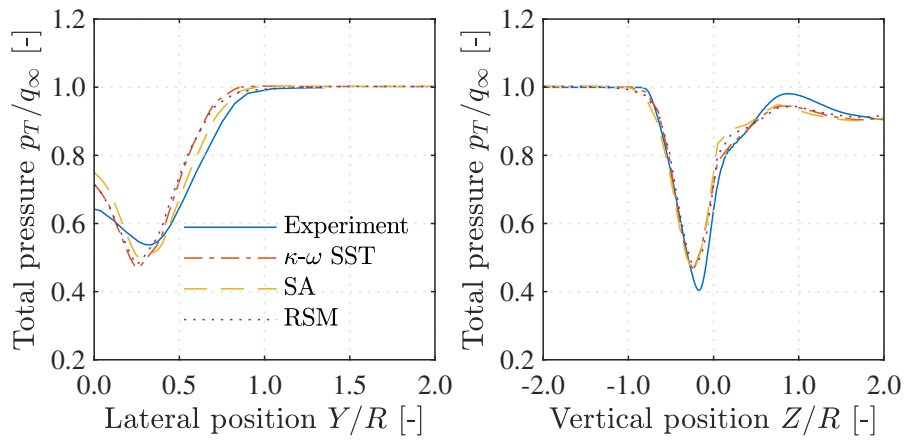

Figure 4: Total pressure profiles along the vertical and lateral radial directions measured in the wake of the model.

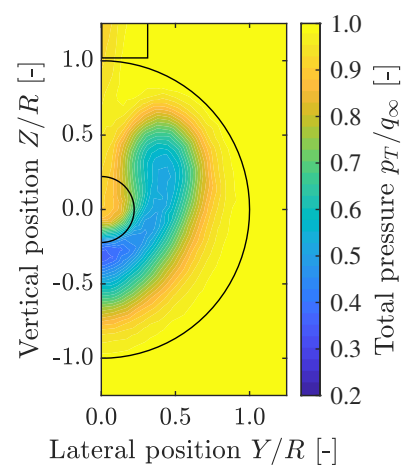

(a) Baseline.

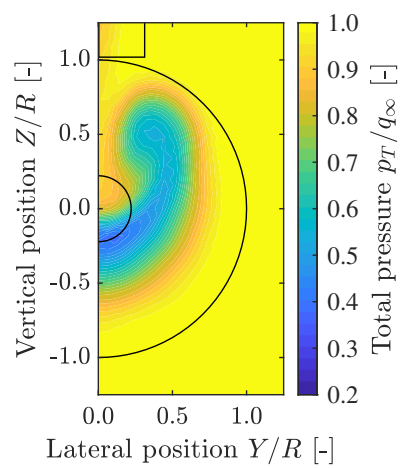

(c) Elliptical LE.

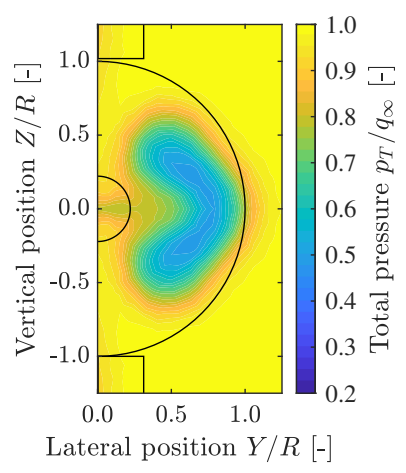

(b) Baseline, mirrored.

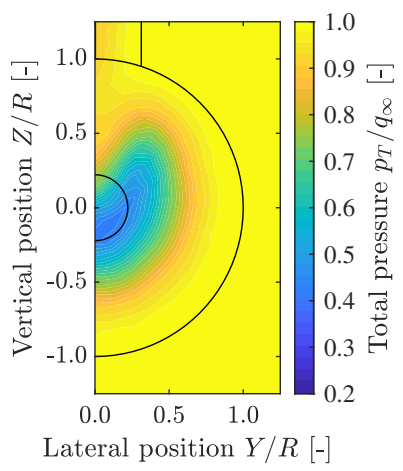

(d) Elliptical LE, curved tip.

Figure 5: Total pressure distribution at $X_{T E}=1.875 R$ (CFD results). 


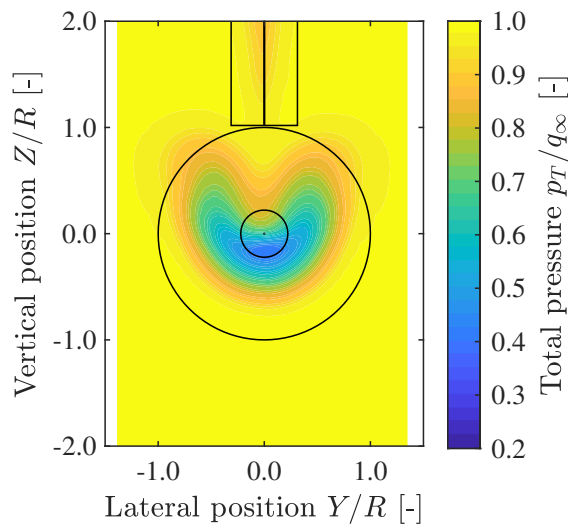

(a) Baseline configuration, wake contour.

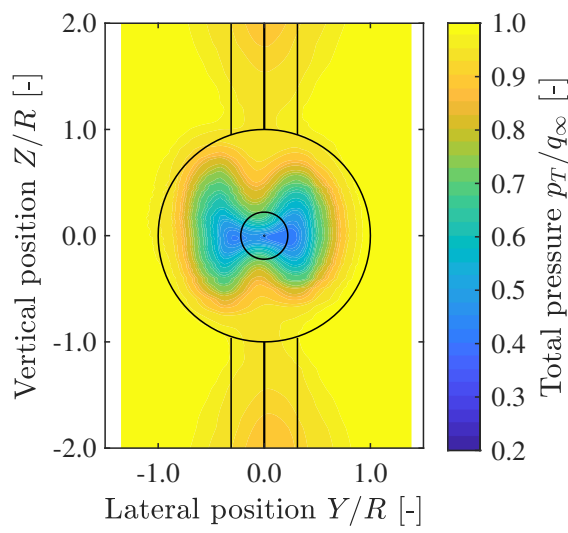

(c) Optimized configuration, wake contour.

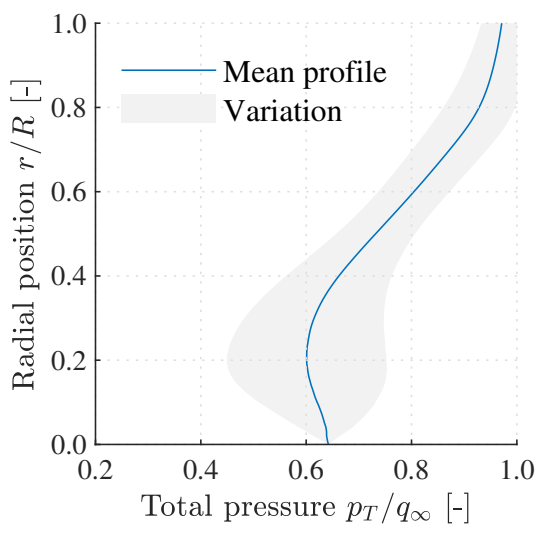

(b) Baseline configuration, mean radial profile.

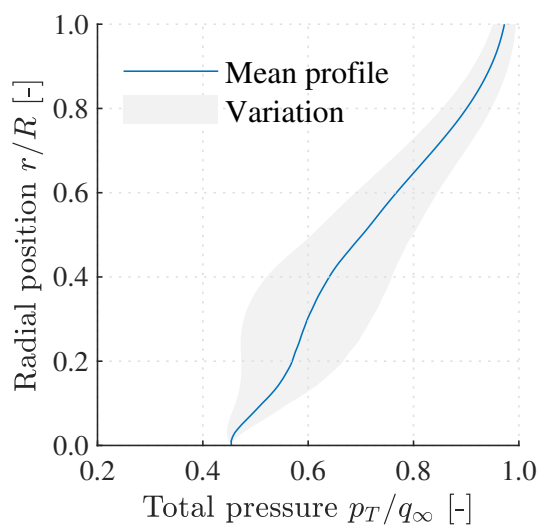

(d) Optimized configuration, mean radial profile

Figure 6: Total pressure distribution in the wake of the model (experimental results).

\subsection{Experimental results}

The baseline and optimized configurations sketched in Figures $1 \mathrm{~b}$ and $1 \mathrm{c}$ were tested in a wind-tunnel campaign. The results of the measurements are reported in Figure 6, in terms of wake total-pressure ratio distributions.

Similarly to what obtained by the CFD analysis, the $p_{T}$ contour for the baseline configuration (Fig 6a) shows a strongly asymmetric distribution around the horizontal plane $(Z / L=0)$. In particular, on the upper side $(Z / L>0)$ it is evident the effect of the horseshoe vortex which entrains high-momentum flow inside the fuselage boundary layer. It is also confirmed that the vortex produces a vertical displacement of the fuselage wake toward the bottom. In fact, the minimum total pressure is not found at the location of the trailing edge but slightly below. The plot of Figure $6 \mathrm{~b}$ displays the mean radial $p_{T}$ profile and its standard deviation obtained by averaging out the profiles at various azimuth angles. The mean profile features a double curvature due to the vortex-induced entrainment. The maximum variation is equal to $0.15 q_{\infty}$.

In addition, due to the implementation of the mirrored fairing on the bottom of the setup (see Fig. 1), also the horizontal plane is a symmetry plane. This condition has a direct effect on the flow development in the wake of the model, as the downward displacement of the wake measured for the baseline configuration is now completely avoided. As a result, the center of 
the wake (i.e. the location of the minimum total pressure) remains on the fuselage axis. The interaction with the vortex also shows improvements over the baseline configuration. Due to the addition of the leading-edge fillet, the pressure gradient acting on the boundary layer at the wing-junction location is reduced. Consequently, the strength of the vortex forming at the fairing leading edge is lower and the wake contour shows a less pronounced footprint when compared to the baseline configuration. The mean radial profile shown in Figure 6d displays a maximum variation of $0.12 q_{\infty}$, meaning a reduction of $20 \%$ over the baseline configuration.

\section{Conclusions and future work}

This paper presented the numerical and experimental analysis on the aerodynamic interaction taking place between the support structure and fuselage body in a wind-tunnel setup developed to study boundary layer ingestion applications. Numerical simulations were carried out to design the fairing around the support strut with the aim of mitigating its aerodynamic interactions with the fuselage boundary layer. CFD analysis showed that the addition of a dummy fairing diametrically opposed to the first one and leading-edge fillets reduced the strength of the interaction. Subsequent experimental measurements on the optimized configuration confirmed the trends observed with the numerical analysis. Future work will focus on the experimental analysis of a powered configuration, where a scaled fan model will be installed on the fuselage tail in an integrated configuration.

\section{Acknowledgments}

This work was conducted within the CENTRELINE project, which has received funding from the European Union's Horizon 2020 research and innovation programme under Grant Agreement No. 723242.

\section{References}

[1] B.J.C. Horsten, Ph.D. thesis, Delft University of Technology (2011)

[2] R.L. Simpson, Annual Review of Fluid Mechanics 33, 415 (2001)

[3] J.L. Fleming, R. Simpson, J. Cowling, W. Devenport, Experiments in fluids 14, 366 (1993)

[4] F. Gand, S. Deck, V. Brunet, P. Sagaut, Physics of Fluids 22, 115111 (2010)

[5] F. Gand, V. Brunet, S. Deck, AIAA journal 50, 2711 (2012)

[6] B.C. Hinson, K.A. Hoffmann, Journal of Aircraft 52, 1492 (2015)

[7] B.E. Green, J.L. Whitesides, Journal of Aircraft 40, 282 (2003)

[8] B.W. Van Oudheusden, C.B. Steenaert, L.M. Boermans, Journal of Aircraft 41, 238 (2004)

[9] A. Seitz, F. Peter, J. Bijewitz, A. Habermann, Z. Goraj, M. Kowalski, A.C. Pardo, C. Hall, F. Meller, R. Merkler et al., 31st Congress of the International Council of the Aeronautical Sciences pp. 09-14 (2018)

[10] Z. Belligoli, A.J. Koers, R.P. Dwight, G. Eitelberg, AIAA Journal 57, 1468 (2018)

[11] P. Spalart, S. Allmaras, 30th aerospace sciences meeting and exhibit p. 439 (1992)

[12] F.R. Menter, AIAA journal 32, 1598 (1994)

[13] D.C. Wilcox et al., Turbulence modeling for CFD, Vol. 2 (La Canada, 1998) 